\title{
Factors Associated with Deep Surgical Site Infection in Spinal Surgery
}

Ed Simor Khan Mor Japar Khan ${ }^{1}$, Kow Ren $\mathrm{Yi}^{2}$, Khairul Bariyyah Binti Mohamad Arifin $^{2}$, Colin Komahen ${ }^{2}$, Low Chooi Leng ${ }^{1}$

${ }^{1}$ Department of Orthopaedics, Traumatology and Rehabilitation, Kulliyyah of Medicine, International Islamic University Malaysia

${ }^{2}$ Department of Orthopaedics, Hospital Tengku Ampuan Afzan

\section{Presenter: Kow Ren Yi}

Introduction: Surgical site infection (SSI) rate in spinal surgery ranges from $1 \%$ to $9 \%$, depending on the type of procedure and institution. SSI gives rise to increased morbidity, poorer outcomes and increased healthcare costs. Various risk factors have been reported in the literature but there is no such related report from Malaysia. This pilot study aimed to determine the incidence and risk factors of deep surgical site infections which require surgical debridement in patients who had undergone spinal surgeries. Materials and Methods: Patients who had undergone spinal surgery at Hospital Tengku Ampuan Afzan, Kuantan from the $1^{\text {st }}$ January 2016 to the $31^{\text {st }}$ December 2017 were included in this study. Associations between SSI and risk factors were analysed with IBM SPSS version 21. Age, body mass index, number of vertebral level involvement, hemoglobin reduction and white blood cell count were analysed by the student t-test while gender, smoking status, spinal cord involvement, fracture dislocation at thoraco-lumbar junction and history of pre-operative blood product transfusion were analysed by Fisher's exact test. Results: Four (17\%) out of 24 patients developed deep SSI which required surgical debridement. Fracture dislocation at the thoraco-lumbar junction $(p=0.008)$ and history of pre-operative blood product transfusion $(p=0.003)$ were associated with deep SSI. Conclusions: This study highlighted different risk factors associated with deep SSI in spinal surgeries. A larger study is needed to further confirm these findings. 